\title{
Interstitial Lung Disease Following Single-Agent Nanoparticle Albumin-Bound Paclitaxel Treatment in Patients with Advanced Non-Small Cell Lung Cancer
}

\author{
Yuki Nakatani Aya Nakaya Takayasu Kurata Takashi Yokoi \\ Yuki Takeyasu Maiko Niki Kayoko Kibata Naoko Satsutani \\ Makoto Ogata Takayuki Miyara Shosaku Nomura \\ First Department of Internal Medicine, Kansai Medical University, Hirakata, Japan
}

\section{Keywords}

Interstitial lung disease $\cdot$ Nanoparticle albumin-bound paclitaxel · Non-small cell lung cancer . Single-agent treatment

\begin{abstract}
Interstitial lung disease (ILD) is a serious and potentially fatal adverse event in lung cancer therapy. Nanoparticle albumin-bound paclitaxel (nab-PTX) is a novel, solvent-free formulation of paclitaxel (PTX). Although the incidence of nab-PTX-induced ILD is not clear, it is generally considered that this formulation presents a similar risk of developing ILD as PTX. Here, we report 3 patients who developed severe ILD following treatment with nab-PTX. We draw attention to the risk of developing drug-induced ILD following nab-PTX treatment, and highlight that this novel formulation might therefore not be as safe as PTX with respect to the development of ILD.


 Oncology}

\section{Introduction}

Nanoparticle albumin-bound paclitaxel (nab-PTX) is an albumin-bound formulation of paclitaxel (PTX). In first-line therapy for patients with advanced non-small cell lung cancer (NSCLC), nab-PTX in combination with other platinum reagents showed significant efficacy in improving patients' prognosis as well as reducing toxicity profiles [1-3]. PTX-induced interstitial lung disease (ILD) occurs in approximately $1 \%$ of treated patients $[4,5]$; thus, PTX is considered relatively safety with respect to the risk of developing drug-induced ILD. Although the incidence of nab-PTX-induced ILD is not clear, it is generally thought that this formulation presents a similar risk of developing ILD as PTX.

To date, only one study has evaluated the development of nab-PTX-induced ILD in clinical trials [6]. Here, we present case reports of 3 patients who developed severe ILD following treatment with nab-PTX. Our reports highlight the risk of developing drug-induced ILD following nab-PTX therapy and indicate that this novel formulation might therefore not be as safe as PTX with respect to the development of ILD.

\section{Case Reports}

Case 1

A 71-year-old male ex-smoker, who was diagnosed with stage IV NSCLC (adenocarcinoma), received a platinum-based doublet regimen (carboplatin, pemetrexed, and bevacizumab) as initial chemotherapy until progression. nab-PTX $\left(100 \mathrm{mg} / \mathrm{m}^{2}\right)$ was subsequently used as a second-line regimen. On day 23 of the second cycle of nab-PTX treatment, he developed grade 1 fever and grade 3 dyspnea. Computed tomography (CT) scanning revealed hyperplasia of interlobular septa, nodular shadows, and ground glass opacity, which suggested a pattern of hypersensitivity pneumonia (Fig. 1). There was no elevation of tumor markers and the size of the target lesion was stable. Cultures of sputum, blood, and mycobacterium were all negative. The results of serological testing for Candida antigen and Aspergillus antigen were negative. $(1 \rightarrow 3) \beta$-D-glucan was not elevated. Although all infectious disease tests were negative, and antibiotics were started prophylactically (tazobactam/piperacillin $13.5 \mathrm{~g} /$ day). Serum KL-6 level was $985 \mathrm{U} / \mathrm{mL}$. Since the respiratory condition had deteriorated, bronchoscopy could not be performed. Clinically, we diagnosed nab-PTX-induced ILD. The drug lymphocyte stimulation test (DLST) was not conducted. Steroid treatment (prednisolone $0.5 \mathrm{mg} / \mathrm{kg}$ ) was immediately initiated and the ILD symptoms quickly improved. Then steroid treatment was decreased. As there was no alternative anticancer agent for lung cancer, a minimum dose for the remainder of nab-PTX treatment was started. We explained the risk of exacerbation of ILD and obtained the patient's consent. nab-PTX treatment was continued for two further cycles until progression, and the ILD was not exacerbated during the remaining treatment.

Case 2

A 74-year-old male ex-smoker, who was diagnosed with stage IV NSCLC (squamous carcinoma), received a platinum-based doublet regimen (carboplatin, tegafur/ gimeracil/oteracil) as initial chemotherapy and nab-PTX $\left(100 \mathrm{mg} / \mathrm{m}^{2}\right)$ as a second-line regimen. On day 24 of the first cycle of nab-PTX, he developed grade 1 fever and grade 3 dys pnea. There was no elevation of tumor markers and the size of the target lesion was stable. Cultures of sputum, blood, and mycobacterium were all negative. The results of serological 


\section{Case Reports in Oncology}

testing for Candida antigen and Aspergillus antigen were negative. $(1 \rightarrow 3) \beta$-D-glucan was not elevated. Although all infectious disease tests were negative, antibiotics were started prophylactically (meropenem $3 \mathrm{~g} /$ day). Serum KL-6 level was $334 \mathrm{U} / \mathrm{ml}$. Since the respiratory condition had deteriorated, bronchoscopy could not be performed. CT scanning showed scattered ground glass opacity and hyperplasia of bronchovascular bundles, which suggested a pattern of hypersensitivity pneumonia (Fig. 2). Clinically, we diagnosed nab-PTXinduced ILD. DLST was not conducted. He was immediately given steroid treatment (prednisolone $0.5 \mathrm{mg} / \mathrm{kg}$ ). ILD improved and steroid treatment was decreased to a maintenance level. As there was no alternative anticancer agent for lung cancer, a minimum dose of nabPTX treatment was started. We explained the risk of exacerbation of ILD and obtained patient's consent. nab-PTX was continued for two further cycles until progression. During the remaining nab-PTX treatment, the patient's ILD did not worsen.

Case 3

A 59-year-old male ex-smoker, whose disease had relapsed following surgery for stage IIIB NSCLC (adenocarcinoma), was treated with a platinum-based doublet regimen (cisplatin and vinorelbine) and concurrent radiation therapy as a first-line regimen, followed by another platinum-based doublet regimen (carboplatin, pemetrexed, and bevacizumab); nabPTX was subsequently given as a third-line regimen. On day 24 of the second cycle of nabPTX therapy, the patient developed grade 1 fever and grade 3 dyspnea. CT scanning revealed consolidation with an air bronchogram and interlobular ground glass opacity, which suggested a pattern of organizing pneumonia (Fig. 3). There was no elevation of tumor markers and the size of the target lesion was stable. Cultures of sputum, blood, and mycobacterium were all negative. The results of serological testing for Candida antigen and Aspergillus antigen were negative. $(1 \rightarrow 3) \beta$-D-glucan was not elevated. Although all infectious disease tests were negative, antibiotics were started prophylactically (tazobactam/piperacillin 13.5 $\mathrm{g} /$ day). Since the respiratory condition had deteriorated, bronchoscopy could not be performed. Clinically, we diagnosed nab-PTX-induced ILD. DLST was not conducted. Steroid treatment (prednisolone $0.5 \mathrm{mg} / \mathrm{kg}$ ) was immediately initiated, and ILD temporarily improved. nab-PTX treatment was discontinued and a minimum dose of steroid was maintained; however, the patient's condition worsened and he developed infectious pneumonia. Despite steroid pulse therapy, the patient died of respiratory failure approximately 6 months after developing ILD.

\section{Discussion}

ILD is a serious and potentially fatal condition associated with lung cancer therapy and its development has been associated with treatment with several anti-cancer agents. Given that PTX-induced ILD occurs in approximately $1 \%$ of treated patients $[4,5]$, this drug is considered to present a low risk of development of ILD. nab-PTX is a novel, solvent-free formulation of PTX that shows significant efficacy in improving patients' prognosis, with a reduced toxicity profile, when administered in combination with carboplatin. However, the risk of developing ILD following nab-PTX treatment remains largely unknown.

No incidence of ILD was reported as an adverse event in the largest phase III clinical trial of nab-PTX in patients with NSCLC conducted to date [1]. Furthermore, a phase I/II study of nab-PTX as a first-line single agent did not report any adverse events related to ILD [7]. Two phase II studies have investigated nab-PTX as a second-line regimen $[6,8]$. In one of the 
phase II studies, 2 patients developed ILD but neither case was fatal. According to our case reports, although the number of cases was too small to be conclusive, male gender and exsmoker status might represent risk factors for developing ILD. One patient underwent radiation therapy, although the relevance of a relationship between radiation therapy and the onset of ILD is unclear. Furthermore, an investigation of the risk of administering nab-PTX therapy to lung cancer patients with a history of ILD would be beneficial.

The present study was limited in size and data were collected retrospectively. Additionally, no bronchoalveolar lavage or lung biopsy were performed to confirm ILD. Each pattern was evaluated using only CT.

Although the incidence of ILD is relatively low, it might be premature to consider nabPTX as having a similar safety profile to PTX with respect to the development of this condition. Therefore, further studies to investigate the relationship between nab-PTX and ILD are warranted.

\section{Statement of Ethics}

The material in the manuscript has been acquired according to modern ethical standards and written informed consent of the patients has been obtained.

\section{Disclosure Statement}

None of the authors have any conflict of interest to declare.

\section{Author Contributions}

All authors contributed equally to the literature search, data collection (including figures), and manuscript writing.

\section{References}

1 Socinski MA, Bondarenko I, Karaseva NA, et al: Weekly nab-paclitaxel in combination with carboplatin versus solvent-based paclitaxel plus carboplatin as first-line therapy in patients with advanced nonsmall-cell lung cancer: final results of a phase III trial. J Clin Oncol 2012;30:2055-2062.

-2 Socinski MA, Okamoto I, Hon JK, et al: Safety and efficacy analysis by histology of weekly nab-paclitaxel in combination with carboplatin as first-line therapy in patients with advanced non-small-cell lung cancer. Ann Oncol 2013;24:2390-2396.

-3 Fang Y, Wang L, Xia GH, Shi MQ: Clinical investigation of efficacy of albumin bound paclitaxel plus platinum compounds as first-line chemotherapy for stage III/IV squamous non-small cell lung cancer. Asian Pac J Cancer Prev 2014;15:7453-7457.

4 Khan A, McNally D, Tutschka PJ, Bilgrami S: Phase II trial of weekly paclitaxel in previously untreated advanced non-small-cell lung cancer. Oncology 2003;65:224-228.

-5 Yasuda K, Igishi T, Kawasaki Y, Yamamoto M, Kato K, Matsumoto S, Kotani M, Sako T, Shigeoka Y, Sugitani A, Histuda Y, Shimizu E: Paclitaxel-induced acute bilateral pneumonitis. Ann Pharmacother 1997;31:1471-1474.

6 Sakata S, Saeki S, Okamoto I, et al: Phase II trial of weekly nab-paclitaxel for previously treated advanced non-small cell lung cancer: Kumamoto thoracic oncology study group (KTOSG) trial 1301. Lung Cancer 2016;99:41-45. 


\section{Case Reports in Oncology}

7 Rizvi NA, Riely GJ, Azzoli CG, et al: Phase I/II trial of weekly intravenous 130-nm albumin-bound paclitaxel as initial chemotherapy in patients with stage IV non-small-cell lung cancer. J Clin Oncol 2008;26:639-643.

8 Hu W, Zhang Z: A phase II clinical study of using nab-paclitaxel as second-line chemotherapy for Chinese patients with advanced non-small cell lung cancer. Med Oncol 2015;32:177.

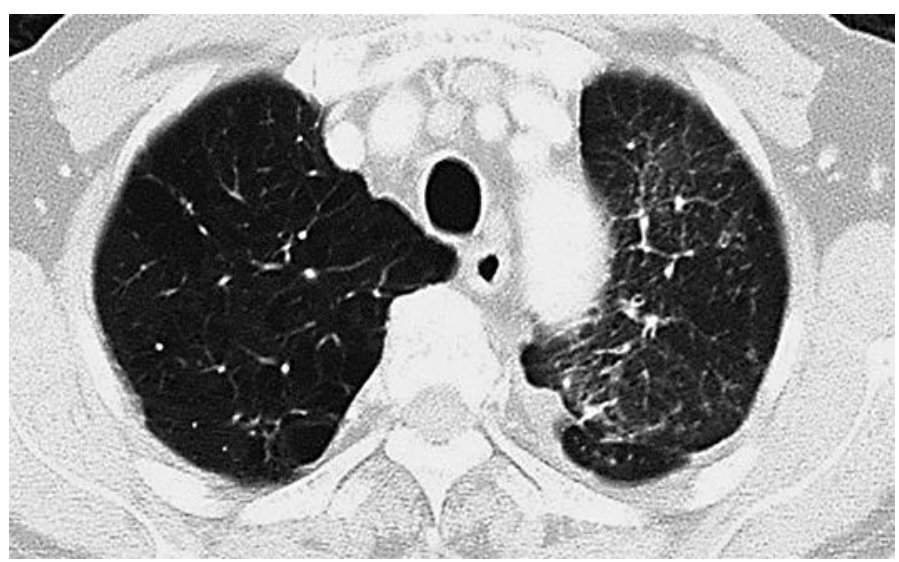

Fig. 1. Plain chest computed tomography of case 1 . Hyperplasia of interlobular septa, nodular shadows, and ground glass opacity, which suggest a pattern of hypersensitivity pneumonia.

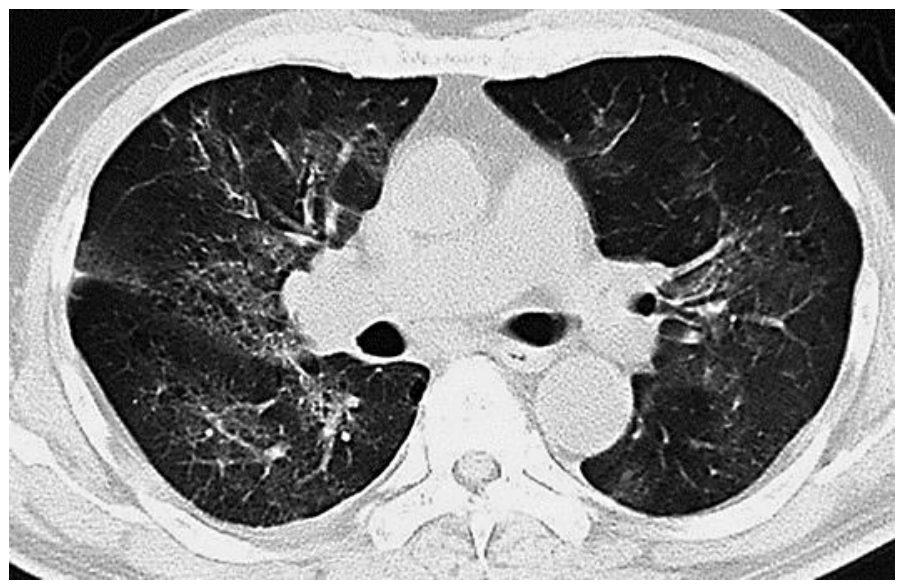

Fig. 2. Plain chest computed tomography of case 2. Scattered ground glass opacity and hyperplasia of bronchovascular bundles, which suggest a pattern of hypersensitivity pneumonia. 


\section{Case Reports in Oncology}

Nakatani et al: Interstitial Lung Disease Following Single-Agent Nanoparticle AlbuminBound Paclitaxel Treatment in Patients with Advanced Non-Small Cell Lung Cancer

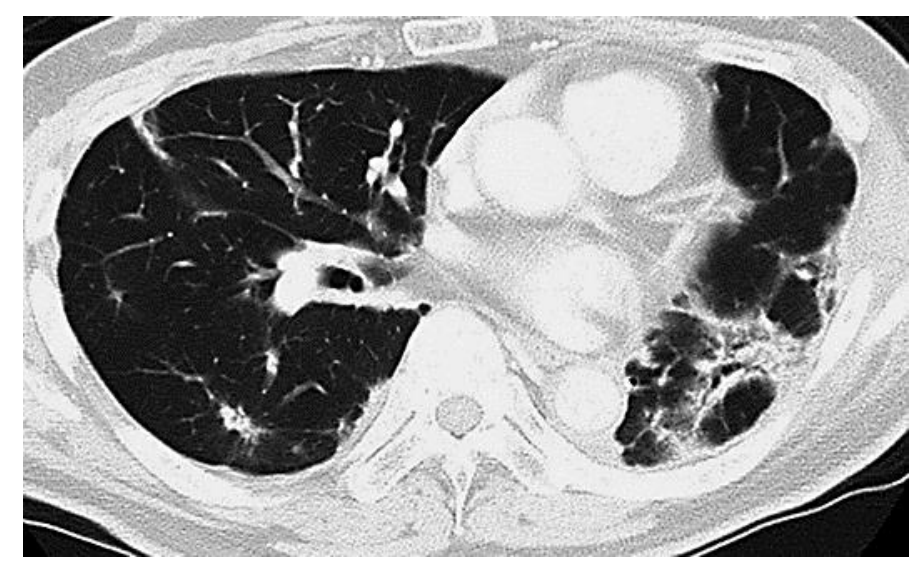

Fig. 3. Plain chest computed tomography of case 3. Consolidation with an air bronchogram and interlobular ground glass opacity, which suggest a pattern of organizing pneumonia. 